\title{
Hamartoma congénito simple del epitelio pigmentado de la retina. Hallazgo casual
}

\section{Congenital simple hamartoma of the retinal pigment epithelium. Casual finding}

\author{
Ana Belén González-Escobar ${ }^{1 *}$, Antonio Ramos-Suárez², Francisco José Barrero-Sojo, \\ Mercedes Lorenzo-Soto ${ }^{3}$, Saturnino Gismero-Moreno ${ }^{1}$

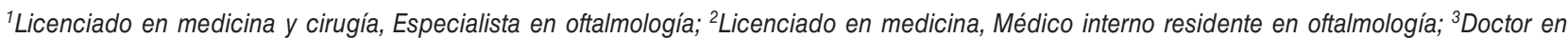 \\ medicina, Especialista en oftalmología. Departamento de Oftalmología, Hospital Costa del Sol, Marbella, Málaga, España
}

\section{Resumen}

Introducción: El hamartoma congénito simple del epitelio pigmentado de la retina (HCSEPR) es un tumor benigno, unilateral y solitario, que suele localizarse cerca de la mácula adyacente a la fóvea, y permanecer invariable a lo largo de la vida. Caso clínico: Paciente de 62 años de edad diagnosticado por la clínica y por la tomografía de coherencia óptica (OCT) de (HCSEPR) descubierto en una exploración rutinaria oftalmológica. Permanece sin cambios en las sucesivas revisiones. Discusión: Debe realizarse un correcto diagnóstico diferencial de la lesión con otras de carácter maligno y un correcto seguimiento, para su despistaje de posibles complicaciones entre ellas la exudación y contracción retiniana. Conclusión: El diagnóstico diferencial de los HCSEPR con otro tipo de tumores es muy importante, para ello nos basamos, sobre todo en la OCT.

Palabras clave: Hamartoma congénito simple. Epitelio pigmentario retiniano. Tumor benigno retiniano. Diagnóstico diferencial. Tomografía de coherencia óptica.

\begin{abstract}
Introduction: The simple congenital hamartoma of the retinal pigment epithelium (HCSEPR) is a benign, unilateral and solitary tumor, which is usually located near the macula, adjacent to the fovea, and remain unchanged throughout life. Case Report: A 62-year-old is diagnosed by clinical symptoms and optical coherence tomography (OCT) of (CSHRPE) discovered in a routine eye examination. Remains unchanged in subsequent revisions. Discussion: correct differential diagnosis of malignant lesion with other character should be performed, and proper follow up for screening of possible complications including exudation and retinal contraction. Conclusion: The differential diagnosis of CSHRPE with other types of tumors is very important, for this we rely primarily on OCT.
\end{abstract}

Key words: Congenital simple hamartoma. Retinal pigment epithelium. Retinal benign tumor. Differential diagnosis. Tomography optical coherence. 


\section{Introducción}

El hamartoma congénito simple del epitelio pigmentado de la retina (HCSEPR) es un tumor benigno, unilateral y solitario, que suele localizarse cerca de la mácula adyacente a la fóvea, con un diámetro menor de $1 \mathrm{~mm}$ y de 1-2,5 mm de grosor. En algunos casos el nódulo puede protruir a través de la retina neurosensorial, alcanzando la cavidad vítrea. Es muy poco común, y hay muy pocos casos descritos en la literatura. Fue descrito inicialmente por Laqua en 1981 y, posteriormente caracterizado y nombrado por Gass en 1989 que informo de 10 casos. En 2003 Shields también informó de una serie extensa de casos. Solo uno de los 15 pacientes vistos por Gass y Shields presentó una mala agudeza visual $(\mathrm{AV})^{1,2}$. Está compuesto casi exclusivamente por células del epitelio pigmentario retiniano y su origen es congénito. Suelen permanecer invariables a lo largo de la vida, y no suelen malignizar. A menudo se asocian a leve tracción adyacente ( $80 \%$ de los casos), exudación retiniana ( $20 \%$ de los casos) y células pigmentadas en cavidad vítrea ( $20 \%$ de los casos). Tienen arteria y vena nutricia mínimamente dilatadas $\left(100 \%\right.$ de los casos) ${ }^{3}$.

\section{Caso clínico}

Paciente de 62 años, acude remitido por su médico de familia por detectar en retinografía para despistaje de retinopatía diabética una lesión pigmentada en el ojo derecho. A la exploración presenta una AV de 0,7 en el ojo derecho y 0,6 en el ojo izquierdo. La presión intraocular de ambos ojos está dentro de la normalidad. A la biomicroscopía, presenta cataratas corticales en ambos ojos. En la funduscopia presenta una lesión nasal a la papila muy pigmentada, de pequeño tamaño y sobreelevada en el ojo derecho, sin evidencia de edema, tracción, exudación o líquido subretiniano (Fig. 1). La funduscopia del ojo izquierdo era normal. Se realiza autofluorescencia (AF), donde se aprecia hipoautofluorescencia en el área correspondiente a la lesión (Fig. 2). La tomografía de coherencia óptica (OCT) realizada en la lesión nos deja ver una hiperreflectividad en capas internas con sombra posterior (Fig. 1). Una vez diagnosticado de HCSEPR por la clínica y los hallazgos tomográficos, se revisa al paciente periódicamente sin percibir ningún cambio en la lesión.

\section{Discusión}

EI HCSEPR es un tumor benigno que se suele diagnosticar incidentalmente. Es un tumor raro que se puede

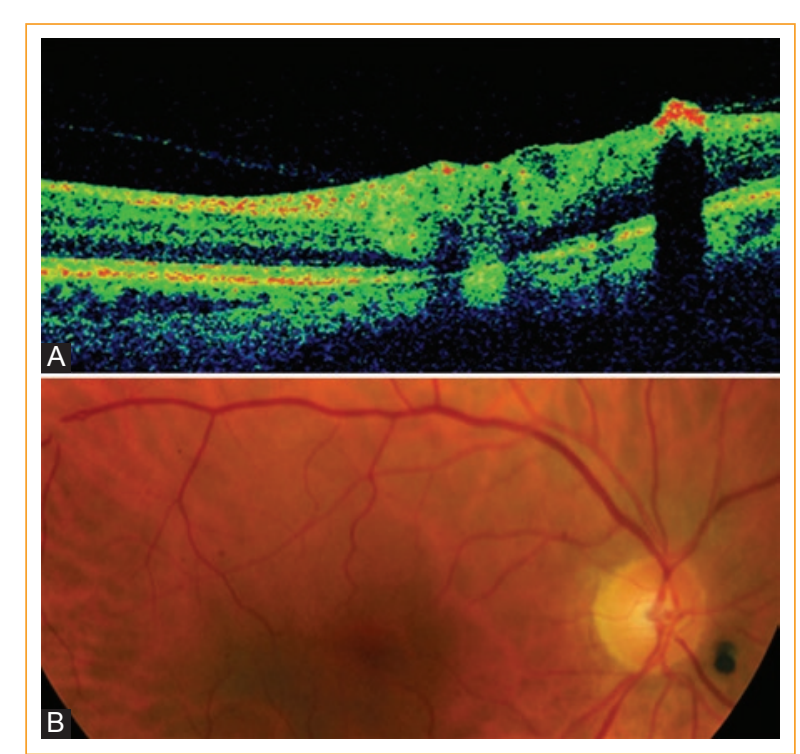

Figura 1. OCT donde apreciamos lesión hiperreflectiva encapas internas con sombra posterior (A). Retinografía (B).

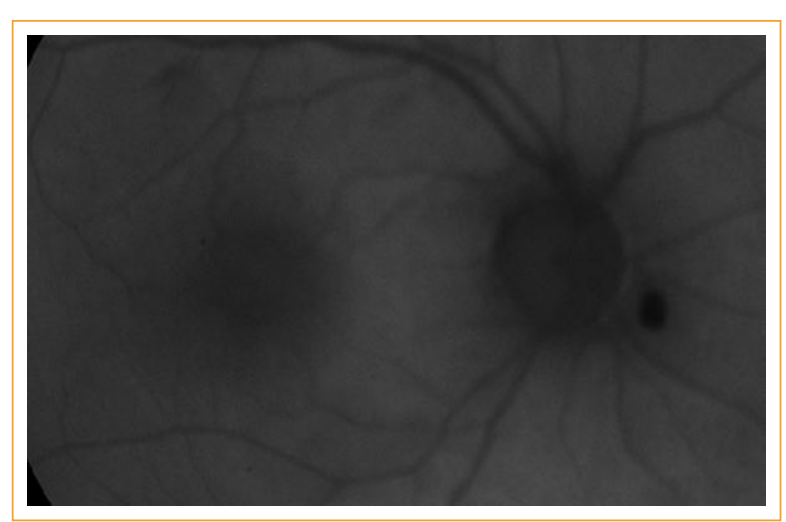

Figura 2. Autofluorescencia con imagen

hipoautofluorescentede la lesión nasal a papila.

detectar durante el examen de fondo de ojo ocular de rutina. En la exploración clínica aparece como una lesión solitaria, pigmentada y bien definida, que envuelve todas las capas de la retina ${ }^{1}$. Puede estar asociado a tracción retiniana, exudación y células pigmentadas en vítreo ${ }^{4}$. El diagnóstico diferencial incluye el hamartoma combinado de la retina y del epitelio pigmentario retiniano (EPR) (lesiones retinianas de grosor completo de color gris marengo con tortuosidad vascular y membranas prerretinianas asociadas); la hipertrofia congénita del EPR (lesiones planas limitadas al plano del EPR, sin invasión retiniana); adenoma o adenocarcinoma del EPR (son tumores adquiridos, diagnosticados en pacientes 
mayores que se comportan de forma diferente, mostrando crecimiento progresivo, exudación, fluido subrretiniano y hemovítreo); la hiperplasia del EPR (pueden desarrollarse hasta convertirse en una masa retiniana de espesor completo, aunque generalmente se observa un moteado disperso del EPR adyacente, y evidencia de la causa previa que ha inducido la hiperplasia); cuerpo extraño intraocular (asociado a historia de traumatismo ocular, evidencia focos de fibrosis y tracción vítrea, mostrando un patrón de sombra acústica posterior en la ecografía); nevus y melanoma coroideos; membranas epirretinianas, etc. ${ }^{1,3,5,6}$. Todo ello se puede descartar por los exámenes complementarios. La ecografía ocular muestra una masa nodular ecogénica de moderada a alta reflectividad interna, sin sombra. La angiografía con fluoresceína muestra una lesión hipofluorescente durante todo el angiograma, en algunos casos en fases tardías se puede apreciar una hiperfluorescencia de la lesión en anillo. La autofluorescencia muestra una hipoautofluorescencia de la lesión ${ }^{4}$. La OCT es un examen no invasivo que ayuda al diagnóstico, y proporciona información acerca de las características del tumor como la hiperreflectividad de capas internas con sombra posterior ${ }^{1,3}$.

El tratamiento, ya que suelen permanecer invariables a lo largo del tiempo, es la observación. En algún caso con contracción o membrana epirretiniana, se puede practicar una vitrectomía siempre valorando beneficio-riesgo y, en casos de edema macular con pérdida de visión, se puede tratar con inyección intravítrea de fármaco antiangiogénico ${ }^{7}$.

\section{Conclusión}

El diagnóstico diferencial de los HCSEPR con otro tipo de tumores es muy importante, para ello nos basamos, sobre todo en la OCT. Además, debe realizarse un correcto seguimiento de la lesión, para su observación y despistaje de posibles complicaciones.

\section{Responsabilidades éticas}

Protección de personas y animales. Los autores declaran que para esta investigación no se han realizado experimentos en seres humanos ni en animales.

Confidencialidad de los datos. Los autores declaran que en este artículo no aparecen datos de pacientes.

Derecho a la privacidad y consentimiento informado. Los autores declaran que en este artículo no aparecen datos de pacientes.

\section{Financiamiento}

Los autores no recibieron patrocinio para llevar a cabo este artículo.

\section{Conflicto de intereses}

Los autores declaran no tener ningún conflicto de intereses.

\section{Bibliografía}

1. Rossi M, Ferreira V, Arana LA, et al. Congenital simple hamar-toma of the retinal pigment epithelium: A case report. Arq Bras Oftalmol. 2014;77:114-5.

2. Barnes AC, Goldman DR, Laver NV et al. Congenital simplehamartoma of the retinal pigment epithelium: Clinical, opticalcoherence tomography, and histopathological correlation. Eye. 2014;28:765-6.

3. García J. Guía de lesiones pigmentadas del fondo de ojo. Guías de Práctica Clínica de la SERV. Sociedad Española de Retina y Vitreo. 2012. [consultado Mar 2012]. Disponible en: http://www.serv.es.

4. Teke MY, Ozdal PC, Batioglu F, et al. Congenital simple hamartoma of retinal pigment epithelium: Clinical and imaging findings. Case Rep Ophthalmol Med. 2012;2012:654-502.

5. Shields CL, Shields JA, Marr BP, et al. Congenital simple hamar-toma of the retinal pigment epithelium: A study of five cases. Ophthalmology. 2003;110:1005-11.

6. Shields CL, Manalac J, Das C, et al. Review of spectral domain-enhanced depth imaging optical coherence tomography of tumors of the retina and retinal pigment epithelium in children and adults. Indian $\mathrm{J}$ Ophthalmo. 2015;63:128-32.

7. Bach A, Gold AS, Villegas VM, et al. Simple hamartoma of the retina pigment epithelium with macular edema. Optom Vis Sci. 2015;92:48-50 